\title{
A study on internal iliac artery branching pattern and its clinical significance
}

\author{
Nataraj KM1, Pavan P Havaldar ${ }^{2}$, Sameen $\mathrm{Taz}^{3}$, Shaik Hussain Saheb ${ }^{4}$ \\ ${ }^{1}$ Dr. Nataraj KM, Associate Professor of Surgery, JJM Medical College, Davangere, Karnataka, India, ${ }^{2}$ Dr. Pavan P Havaldar, \\ Associate Professor of Anatomy, Gadag Institute of Medical Sciences, Mallasamudra, Mulgund Road, Gadag, Karnataka, \\ India, ${ }^{3}$ Dr. Sameen Taz, Assistant Professors, Department of Anatomy, Sri Devaraj Urs Medical College, Kolar, Karnataka, \\ India, ${ }^{4}$ Dr. Shaik Hussain Saheb, Assistant Professor of Anatomy, JJM Medical College, Davangere, Karnataka, India.
}

Address for Correspondence:Dr. Shaik Hussain Saheb, Assistant Professor, Department of Anatomy, JJM Medical College, Davangere, Karnataka, India. Email: anatomyshs@gmail.com

\begin{abstract}
Background: The internal iliac artery is the artery of the pelvis. It supplies most of the blood to the pelvic viscera, gluteal region, medial thigh region and perineum. Injuries of internal iliac artery and its severe bleeding are more common which leads to potentially lethal complication in pelvic surgeries. While operating on pelvic organs, the knowledge of internal iliac artery, its branching pattern and its variations is important for surgeons. Objectives: The present study conducted to study the branching pattern of internal iliac artery by dissection method and to study the variations in the branching pattern. Materials and Methods: Dissection of 50 adult human pelvic halves was procured from embalmed cadavers of J.J.M. Medical College and S.S.I.M.S \& R.C, Davangere for the study. Results: The classification of branching pattern of internal iliac artery was based on modified Adachi classification. Out of the 50 specimens studied, Type Ia arrangement was found in $52 \%$ of the specimens, Type III in 34\%, Type IIa and type V was found in $2 \%$ each, Type IV was not found in any of the specimens and $10 \%$ of the specimens could not be classified because of the absence of inferior gluteal artery in them. Conclusion: Adachi Type Ia arrangement was the most frequent finding. The obturator artery took origin most frequently from the anterior division of internal iliac artery. Middle rectal artery was not constant.
\end{abstract}

Key words- Internal iliac artery, Obturator artery, Middle rectal artery, Internal pudendal artery.

\section{Introduction}

The internal iliac artery is the chief arterial source of pelvis and it is internal division of common iliac artery. It supplies most of the blood to the pelvic viscera, namely; rectum, urinary bladder, prostate and seminal vesicle in male, uterus in female and musculoskeletal part of the pelvis. In case of male pelvis it also supplies branches to the, medial thigh region, gluteal region, the perineum including erectile tissues of the penis and the clitoris. Knowledge of internal iliac artery and its branching pattern is not only important for the anatomists but also for surgeons, obstetricians and gynaecologists, urologists, vascular surgeons and radiologists. Bilateral internal iliac artery ligation is an effective lifesaving method to control obstetrical and gynaecological haemorrhage and avoids a hysterectomy. In various surgeries of pelvic organs like rectal malignancies, haemorrhoidectomy, the knowledge of internal iliac artery, its branching pattern and its

Manuscript Received: $22^{\text {nd }}$ May 2017

Reviewed: $1^{\text {st }}$ June 2017

Author Corrected: $9^{\text {th }}$ June 2017

Accepted for Publication: $15^{\text {th }}$ June 2017
Variations is important for surgeons. Intractable haemorrhage during transurethral resection of prostate surgeries can be controlled by ligation of internal iliac artery, where no definitive bleeding point is detectable [1].

Angiographically directed arterial embolisation is very effective in controlling the haemorrhage and now widely practiced because it is a minimally invasive technique. The intentional ligation of internal iliac artery is also done in the treatment of endovascular repair of aortoiliac aneurysms. The iliac crest flap pedicled on the ilio-lumbar artery, a branch of posterior division of internal iliac artery, is being used as a reliable bone flap. A severe and potentially lethal complication in pelvic injuries is arterial bleeding commonly involving the branches of internal iliac artery, namely, the lateral sacral, ilio-lumbar, obturator, vesical and inferior gluteal arteries. Surgeons must also be conscious of unexpected sources of haemorrhage, such as from an aberrant obturator artery 
Original Research Article

while dealing with direct, indirect inguinal, femoral or obturator hernias and take appropriate precautions to avoid injury to these vessels. Vascular variations have always been a subject of controversy as well as curiosity, because of their clinical significance. Hence, the present work was undertaken to study the internal iliac artery and its branching pattern in pelvis, to know the variations, if any, in the arterial tree [2].

Alexis carrel at the beginning of $20^{\text {th }}$ century first described the technique for vascular suturing and anastomosis and successfully performed many organ transplantations in animals. He thus actually opened the way to modern vascular surgery that was before limited to vessels permanent ligation [3]. In older literature internal iliac artery was referred to as the hypogastric artery. The first attempt to group the variations in the origin of the parietal branches of the internal iliac artery into definite patterns was undertaken by Jastschinski [4].

He found that only the vessels in the first category showed sufficient regularity in origin to enable them to be grouped into definite types, of which he described four.

Adachi [5] modified the method slightly, adding a fifth type of variation and included certain sub types, in a study of internal iliac artery and its branches in Japanese subjects. His scheme is as follows,

\section{ADACHI types:}

H - Internal iliac artery, UMB - Umbilical artery, SG - Superior gluteal artery, IG -Inferior gluteal artery,P - Internal pudendal artery.

Type I : The superior gluteal artery arises separately from the internal iliac artery, and the inferior gluteal and internal pudendal vessels are given off by a common trunk. If the latter divides within the pelvis it is considered to be type Ia, where as if the bifurcation occurs below the pelvic floor it is classified as type $\mathrm{Ib}$.

Type II : The superior and the inferior gluteal arteries arise by a common trunk and the internal pudendal vessels separately. In this category, as in the previous one, two subtypes are described.

Type IIa includes those specimens in which the trunk common to the two gluteal arteries divides within the pelvis and type IIb those in which the division occurs outside the pelvis.

Type III : The three branches arise separately from the internal iliac artery.

Type IV : The three arteries arise by a common trunk. The sub typing is based on the sites of origin of the superior gluteal and the internal pudendal arteries from the parent stem.

In type IVa, the trunk first gives rise to the superior gluteal artery before bifurcating into the other two branches. In type IVb, the internal pudendal is the first vessel to spring from the common trunk, which then divides into superior and inferior gluteal arteries.

Type V : The internal pudendal and the superior gluteal arteries arise from a common trunk and the inferior gluteal has a separate origin (Figure 1). The knowledge of internal iliac artery and its branching pattern is very essential in pelvic surgery practice.

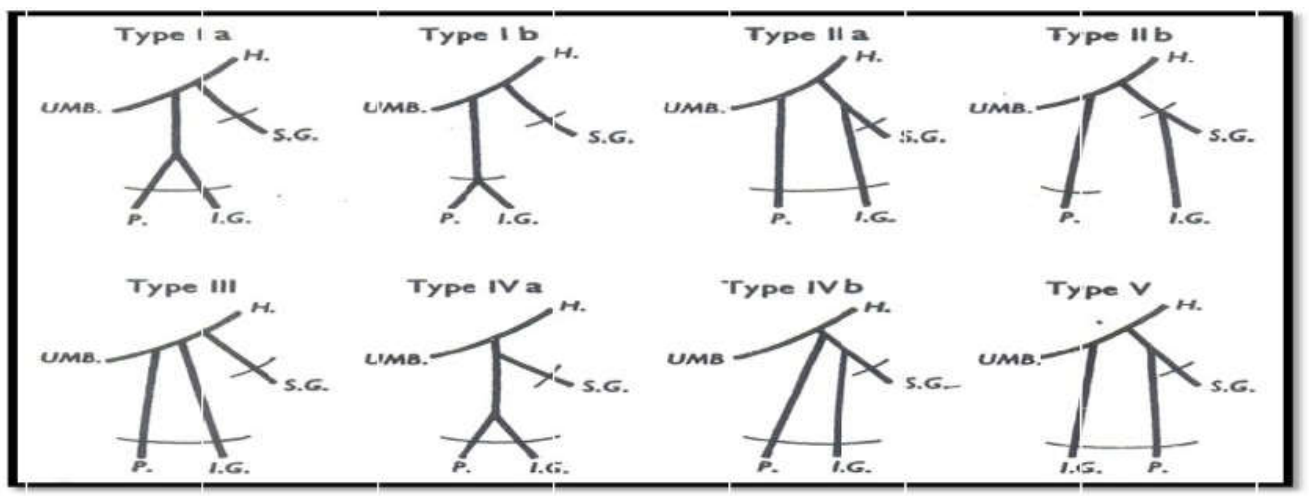

Fig-1: Adachi's types H. internal iliac artery; I.G. Inferior gluteal artery; P. internal pudendal artery; S.G. superior gluteal artery; UMB. umbilical artery 


\section{Materials and Methods}

Study design- Present study designed to study morphological features of uterine and vaginal arteries in human dissected pelvis.

Settings- 50 formalin fixed adult human pelvic halves were procured from the Department of Anatomy, J.J.M. Medical College and S.S. Institute of Medical Sciences and Research Centre, Davangere.

Inclusion criteria- well dissected pelvic sections during routine undergraduate dissection, the specimens with well-preserved vascular pattern.

Exclusion criteria- properly not dissected specimens, spoiled pelvic sections, pelvic sections without or not well preserved vascular pattern.

Methodology- A horizontal section through the abdomen at the fourth lumbar vertebral level was taken. The pelvic specimen thus obtained was divided into two equal halves by cutting through the pubic symphysis, the sacrum and coccyx. This section divided the bladder, (uterus and vagina in female) and rectum longitudinally.

Then, the peritoneum was removed from the bladder, uterus (in female), rectum and the lateral pelvic wall of each half of the pelvis. The level of origin of internal iliac artery was noted, the length of the trunk of the vessel was measured. The level of its termination into anterior and posterior division was identified and noted.

The occasional branches that were arising from the common trunk were dissected. The individual branches (parietal, visceral) arising from the anterior and posterior divisions were dissected upto their terminations inside the pelvis. A pattern of variation that have occurred at the level of origin and division of the main trunk, anamolous branches that have arised from both anterior and posterior divisions, any absence of definitive branches from the anterior and posterior division were noted.

A study of mode of exit of some of the branches outside the pelvic cavity like internal pudendal artery, superior and inferior gluteal artery were also noted.

The specimens were numbered and photographs of each specimen were taken by digital camera and the arterial tree was coloured red digitally and labelled. The specimens were preserved by using $5 \%$ formalin solution.

Statistical analysis- we have calculated simple percentage of uterine and vaginal artery out of total number of specimens.

\section{Results}

In the present study of 50 pelvic halves, the most common site of origin of internal iliac artery was at the level of lumbo-sacral intervertebral disc found in 30 specimens $(60 \%)$. At the level of L5 vertebra internal iliac artery taken origin in $20 \%$ of cases, in $16 \%$ of specimens it taken origin at the level of disc between L4 and L5 and it taken origin at the levels of S1 vertebra in $4 \%$ of cases.

The length of internal iliac artery was found to be $3-5 \mathrm{~cm}$ in 23 specimens $(46 \%), 5-7 \mathrm{~cm}$ in 16 specimens $(32 \%)$ and $1-3 \mathrm{~cm}$ in 11 specimens $(22 \%)$, shortest being $1.5 \mathrm{~cm}$ and longest being $7 \mathrm{~cm}$. The level of division of internal iliac artery took place above the greater sciatic foramen in 34 specimens (68\%), at the upper border of greater sciatic foramen in 7 specimens (14\%), and below the upper border of greater sciatic foramen in 9 specimens (18\%).

The common trunk of internal iliac artery did not give any branch in 22 specimens (44\%), gave origin to vertebral branches in 15 specimens (30\%), to ilio-lumbar artery in 9 specimens $(18 \%)$, superior gluteal artery in 1 specimen $(2 \%)$, lateral sacral artery in 2 specimens (4\%), both iliolumbar artery and lateral sacral artery in 1 specimen (2\%). The obturator artery took origin from the anterior division of internal iliac artery in 36 specimens (72\%). Out of 50 specimens, middle rectal artery took origin from anterior division, alone in2 specimens (4\%), with internal pudendal artery in32 secimens (64\%), with inferior vesical artery in 3 specimens $(6 \%)$, with obturator artery in 1 specimen (2\%), with inferior gluteal artery in 4 specimens $(8 \%)$ and was found to be absent in 8 specimens $(16 \%)$.

The internal pudendal artery took origin from the anterior divisionof internal, alone in 3 specimens $(6 \%)$, along with middle rectal artery in 15 specimens (30\%), with inferior gluteal artery in 25 specimens (50\%), with obturator artery in 3 specimens $(6 \%)$ and with inferior vesical artery in 4 specimens $(8 \%)$. Out of 17 specimens the inferior gluteal artery took origin both from anterior and posterior divisions of internal iliac artery, from the anterior division in in 11 specimens $(22 \%)$ and from posterior division gave rise to inferior gluteal artery directly in 2 specimens $(4 \%)$. Uterine artery took origin from anterior division directly in 15 specimens (88\%). Vaginal artery took origin from anterior division directly in 15 specimens $(88 \%)$.

Out of 50 specimens, ilio-lumbar artery took origin from posterior division, directly in 29 specimens (58\%). From anterior division it took origin in $21(44 \%)$ cases. Superior 


\section{Original Research Article}

gluteal artery took origin from posterior division directly in 44 specimens $(88 \%)$ ). lateral sacral artery took origin from posterior division in 38 specimens $(76 \%)$. From anterior division it originated in $5(10 \%)$ of specimens. The branching pattern of internal iliac artery was classified as per modified Adachi classification. Type Ia arrangement was found in 26 specimens (52\%), Type IIa in 1 specimen (2\%), Type III in 17 specimens (34\%), Type IV arrangement was not found in any of the specimens, Type V was found in 1 specimen $(2 \%)$ and 5 specimens $(10 \%)$ could not be classified, because of the absence of inferior gluteal artery in them.

\section{Discussion}

In the present study, the most common site of origin of internal iliac artery was at the level of lumbo-sacral intervertebral disc and the level of division of internal iliac artery was above the greater sciatic foramen in majority of the specimens. These observations correlate with the observations of Lipschutz [6]. In the present study, the length of internal iliac artery was found to be as short as $1.5 \mathrm{~cm}$ and as long as $7 \mathrm{~cm}$, average length being $3-5 \mathrm{~cm}$ observed in 23 specimens $(46 \%)$.

This correlate with the observations of Bleich AT[7] in which the average length of internal iliac artery was $27 \mathrm{~mm} \&$ Lipschutz [6] $(3.5-4.5 \mathrm{~cm})$. These observations also correlate with the observations of Bergman [8] where the length was found to be as short as $1.2 \mathrm{~cm}$ and as long as $7.5 \mathrm{~cm}$. In the present study, though the basis of classification of branching pattern of internal iliac artery is mainly based on modified Adachi [5] classification, it has been adopted with slight modifications. Adachi [5] studied the branches of internal iliac artery both outside and inside the pelvis and classified the branches into types - Ia, Ib, IIa, IIb, III, IVa, IVb and V.

The present study is confined to the branches of internal iliac artery only inside the pelvis and is confined to types Ia, IIa, III, IVa, IVb and V. In the present study, Type Ia arrangement was found in $52 \%$ of the specimens, Type IIa in $2 \%$, Type III in $34 \%$, Type IV was not found in any of the specimens, Type $\mathrm{V}$ was found in $2 \%$ and $10 \%$ of the specimens could not be classified because of absence of inferior gluteal artery in them.

These observations correlate with the observations made by Braithwaite JL [9] in which Type I arrangement was the most frequent finding, accounting for $58.5 \%$ of all the specimens, Type III in $22.5 \%$, Type II in $15.3 \%$, Type IV pattern was comparatively rare in only $3.6 \%$ of specimens and Type V was not found. In a study conducted on 645 pelvic halves of Japanese cadavers by Yamaki K[10], it was observed that Type I arrangement was most frequently observed in $46.8 \%$ of the specimens. In a study conducted on 167 pelvic halves of Caucasian bodies by Roberts WH [11], it was observed that there were no instances of the rare Type $\mathrm{V}$.

In the present study, obturator artery presented considerable variations in its origin; it was observed that the obturator artery took origin from the anterior division of internal iliac artery in 36 specimens (72\%). Out of which, it took origin as a direct branch in 20 specimens $(40 \%)$ and with other named branches in 16 specimens $(32 \%)$.

These observations correlate with the observations with a previous study, of where it was observed that obturator artery took origin as a direct branch from anterior division (41.4\%) and with other named branches (28.5\%) [8]. It also correlate with the observations of Braithwaite JL[9], where obturator artery took origin as a direct branch from anterior division $(41.4 \%)$ and with other named branches (32\%). Pai MM[2], in which obturator artery took origin from anterior division $(60 \%)$. In the present study, obturator artery took origin from posterior division of internal iliac artery in 9 specimens (18\%). It correlate with the observations of Pai MM(18\%)[2].

This is a slightly higher incidence when compared to the observations of Pick (3.28\%) and Kumar D (0.5\%)[12,13]. In the present study, obturator artery took origin directly from external iliac artery in 1 specimen $(2 \%)$. In the present study, obturator artery took origin from inferior epigastric artery in 3 specimens $(6 \%)$. This is a low incidence when compared with the observations of Bergman (25\%), Braithwaite JL(19.5) [8,9]. Pai MM [2] observed the origin of obturator artery from external iliac artery and inferior epigastric artery in $19 \%$.

In the present study, middle rectal artery took origin from anterior division directly in 2 specimens (4\%), with internal pudendal artery in 3 specimens (64\%), with inferior vesical artery in 3 specimens $(6 \%)$, with obturator artery in 1 specimen (2\%) and with inferior gluteal artery in 4 specimens $(8 \%)$.

Middle rectal artery was found to be absent in 8 specimens $(16 \%)$. It correlate with the observations of Lipshcutz [6] where this vessel was present in only $72 \%$ of the specimens and arises most frequently as a branch of the internal pudendal artery. It also correlate with the observations of Bergman [8], where the middle rectal artery is occasionally absent. It usually arises from internal iliac artery; however, it has been reported as arising from inferior vesical or internal pudendal artery. 


\section{Original Research Article}

In the present study, inferior gluteal artery took origin from anterior division in 42 specimens $(84 \%)$, from posterior division in 3 specimens $(6 \%)$ and was found to be absent in 5 specimens (10\%). The absence of inferior gluteal artery was also observed by Reddy S[14]. In the present study, out of 17 specimens, uterine artery took origin from anterior division directly in 15 specimens $(88 \%)$ and double uterine artery was found in 2 specimens $(12 \%)$.

This correlate with the observations of Bergman [8] in which, the uterine artery usually arises from the internal iliac artery. In the present study, out of 17 specimens, vaginal artery took origin directly from anterior division in 15 specimens $(88 \%)$. Bergman [8] states that, vaginal artery usually arises from the uterine artery, sometimes as several branches, and sometimes from the internal iliac in common with the uterine artery [15].

In the present study, lateral sacral artery took origin from posterior division, paired origin in 38 specimens $(76 \%)$ and unpaired in 7 specimens (14\%). Lateral sacral artery took origin from anterior division with inferior gluteal artery in 2 specimens (4\%) and from common trunk of internal iliac artery in 3 specimens (6\%). Bergman [8] states that, lateral sacral vessels from both sides may arise in common.

The lateral sacrals may provide the inferior vesical and middle rectal arteries. In the present study, the superior vesical artery, inferior vesical artery and superior gluteal artery were fairly constant in their origin and course $[16,17]$

\section{Conclusion}

The knowledge of branching pattern of internal iliac artery may helpful in surgeries in pelvic region specially in obstetrics and gynic practice and treating in diseases of female pelvic organs.

Conflict of interest: None declared. Funding: Nil, Permission from IRB: Yes

\section{References}

1. Moore KL. Clinically oriented anatomy. $4^{\text {th }}$ ed., Baltimore,U.S.A: Williams and Wilkins; 1992.p. 350-55.

2. Pai MM, Krishnamurthy A, Prabhu LV, Pai MV, Kumar SA and Hadimani GA. Variability in the origin of the obturator artery. ClinicsBasic Research 2009; 64 (9): 897-901. doi: 0.1590/ S180759322009000900011.
3. Carrel A. Suture of blood-vessels and transplantation of organs. In: Nobel Lectures, Physiology or Medicine 19011921. Amsterdam: Elsevier Publishing Company, 1967. http:// nobelprize. org/ medicine/ laureates/1912/carrellecture. html. Accessed July 29, 2005.

4. Jastschinski, S. Die typischen Verzweigungsformen Der Arteria hypogastrica. Int. Mschr. Anat. Physiol. 1891 (a): 8;111-127.

5. Adachi, B. Das Arteriensystem der Japaner, Bd. II. Kyoto. Supp. To Acta Scholae Medicinalis Universitatis Imperalis in Kioto, 1928;9:1926-7.

6. Lipschutz B. A composite study of the hypogastric artery and its branches.Ann Surg 1918; 67 (5):584-608.

7. Bleich AT, Rahn DD, Wieslander CK, Wai CY, Roshanravan SM and Corton MM. Posterior division of internal iliac artery: anatomic variations and clinical applications.AmnJ Obstet Gynecol 2007; 197(6): 658. DOI:10.1016/j. ajog. 2007. 08.063

8. Bergman RA, Thompson SA, Afifi AK and Saadeh FA. Compendium of human anatomic variation. Baltimore and Munich: Urban and Schwazenberg; 1988.p.84-85.

9. Braithwaite JL. Variations in origin of the parietal branches of the internal iliac artery. J Anat. 1952 Oct; 86(Pt 4): 423-430. PMCID: PMC1273694.

10. Yamaki K, Saga T, Doi Y, Aida K, Yoshizuka M. A statistical study of the branching of the human internal iliac artery. Kurume Med J. 1998;45(4):333-40.

11. Roberts WH, Krishingner GL. Comparative study of human internal iliac artery based on adachi Classification. The Anatomical Record 2005; 158 (2):191-96. DOI: 10.1002/ar.1091580208.

12. Pick JW, Anson BJ and Ashley FL. The origin of the obturator artery.Am J Anat 1942;70:317-43.

13. Kumar D and Rath G. Anamolous origin of obturator artery from the internal iliac artery - A case report. Int J Morphol 2007;25(3):639-41.

14. Reddy S, Vollala VR and Rao M. Absence of inferior gluteal artery : a rare observation. International Journal of Morphology2007;25(1):95-98. 
15. Havaldar PP, Taz S, Angadi AV, Saheb SH. Morphological study of obturator artery. Int J Anat Res. 2014;2:354-7.

16. Havaldar PP, Taz S, Angadi AV, Saheb SH. Study of posterior division of internal iliac artery. Int $\mathrm{J}$ Anat Res. 2014;2(2):375-9.

17. Pavan $\mathrm{P}$ Havaldar et al. Study of medial circumflex artery.Int J Anat Res.2014;Vol 2(2):380-82.

\section{How to cite this article?}

Nataraj KM, Pavan P Havaldar, Sameen Taz, Shaik Hussain Saheb. A study on internal iliac artery branching pattern and its clinical significance.Int J surg Orthopedics.2017;3(2):44-49.doi:10.17511/ijoso.2017.i02.04. 